\title{
Arbeit in der Industrie 4.0 - Erwartungen des Instituts für angewandte Arbeitswissenschaft e.V.
}

\author{
Klaus-Detlev Becker
}

\section{Wer wir sind}

Das Institut für angewandte Arbeitswissenschaft e.V. (ifaa) ist eine Wissenschaft und Praxis verbindende Institution in den Forschungsdisziplinen Arbeitswissenschaft und Betriebsorganisation. Im Mittelpunkt seiner Arbeit steht die Steigerung der Produktivität in den Unternehmen und damit die Sicherung der Wettbewerbsfähigkeit der deutschen Wirtschaft.

Das ifaa analysiert betriebliche Prozesse, ergründet Wirkzusammenhänge, zeigt Entwicklungen der Arbeits- und Betriebsorganisation auf und erarbeitet unternehmensrelevante praxiserprobte Produkte und Dienstleistungen.

Unter dem Thema „Industrie 4.0“ sammelt das ifaa momentan Beispiele einer weitergehenden Automatisierung und Vernetzung von Betriebsmitteln und wertet die Auswirkungen auf die Beschäftigten aus. Gegenüber den Bestrebungen, die vor einiger Zeit unter dem Stichwort „CIM (computer integrated manufacturing)“ diskutiert wurden, nutzt die „Vision“ Industrie 4.0 die Möglichkeiten des Internets und der mobilen Datengeräte. Das ifaa analysiert und begleitet - in enger Zusammenarbeit mit den Arbeitgeberverbänden den Stand der betrieblichen Umsetzung, fördert den Informationsaustausch zu heranreifenden Fragestellungen und beteiligt sich an der Unterstützung der Unternehmen. Dabei berücksichtigen wir, dass Industrie 4.0 eine längerfristige Entwicklung darstellt, für die es heute erste Ansätze bzgl. der Anwendung in der Praxis gibt, die in der gesamten Breite wie von Experten eingeschätzt - aber voraussichtlich erst in den Jahren 2025/30 zum betrieblichen Alltag gehören wird.

\section{K.-D. Becker $(\varangle)$}

Institut für angewandte Arbeitswissenschaft e.V., Uerdinger Straße 56, 40474 Düsseldorf,

Deutschland

e-mail: k.becker@ifaa-mail.de

(C) The Author(s) 2015

A. Botthof, E.A. Hartmann (Hrsg.), Zukunft der Arbeit in Industrie 4.0,

DOI 10.1007/978-3-662-45915-7_3 


\section{Industrie 4.0 als Chance für die Wettbewerbsfähigkeit von Arbeit}

In Kooperation der drei Industrieverbände BITKOM, VDMA und ZVEI erarbeitete ein „Arbeitskreis Industrie 4.0“ Umsetzungsempfehlungen für das „Zukunftsprojekt Industrie 4.0“. Ziel der daran Beteiligten ist es, diese Entwicklung - ob es sich dabei um eine industrielle Revolution oder Evolution handelt ist zweitrangig - aktiv mitzugestalten und so den Wirtschafsstandort Deutschland zu stärken. Kernelement von Industrie 4.0 ist das Zukunftsbild der „Smart Factory“, in der eine Vielzahl von cyber-physische Systeme zusammenwirken (Kagermann et al. 2013, S. 18). Vereinfacht dargestellt verbinden diese Systeme die vielfältigen Prozesse der Produktion, Logistik, des Engineering, des Managements und der Internetdienste. Sie erfassen mittels Sensoren eigenständig Informationen, tauschen diese über digitale Dienste aus, sind in der Lage auf Grundlage der verarbeiteten Informationen Aktionen auszulösen und sich gegenseitig selbstständig zu steuern. Im Zusammenwirken mit dem Kreativpotential der Beschäftigten lassen sich auf diesem Wege die vielfältigen industriellen Prozesse grundlegend verbessern. Industrie 4.0 leistet darüber hinaus - neben vielen anderen Wirkungsrichtungen - auch einen Beitrag zur Bewältigung der aktuellen Herausforderungen des demografischen Wandels. Insbesondere durch Assistenzsysteme ermöglichen cyber-physische Systeme die Arbeit demografiesensibel und belastungsmindernd zu gestalten. Was unter dem Aspekt des drohenden Fachkräftemangels von wesentlicher Bedeutung sein kann, ist die sich insbesondere durch die Assistenzsysteme entwickelnde Möglichkeit, die Produktivität älterer Arbeitnehmer in einem längeren Arbeitsleben zu erhalten. Monotone oder schwere Tätigkeiten kann die Technik übernehmen, während den Beschäftigten kreative, wertschöpfende Tätigkeiten übertragen werden können, was die Leistungsfähigkeit auf längere Frist erhält. Das heißt nicht, dass künftig nur noch kreative Tätigkeiten seitens der Beschäftigten in den Unternehmen ausgeführt werden. Verringern lässt sich jedoch der Anteil der von Mitarbeitern auszuführenden (körperlich und ggf. auch geistig) belastenden Tätigkeiten. Andererseits wird es auch weiterhin einfache Arbeiten geben. Es ist zu erwarten, dass diese Tätigkeiten durch Industrie 4.0 ebenfalls einen höheren Anteil an Wertschöpfung erbringen können. Das wird dazu beitragen, Arbeit auf unterschiedlichem Anforderungsniveau wettbewerbsfähig zu gestalten und so in Deutschland zu halten.

Erst eine wettbewerbsfähige Arbeit lässt eine flexible Arbeitsorganisation zu, die es den Mitarbeitern, ermöglicht, Beruf und Privatleben sowie Weiterbildung besser miteinander zu kombinieren und so eine Balance zwischen Arbeit und Familie zu erreichen.

Die Autoren der Umsetzungsempfehlungen verweisen darauf, dass auch andere Länder den Trend zur Nutzung des Internets der Dinge und Dienste in der industriellen Produktion erkannt haben (Ebenda).

Um die Wettbewerbsfähigkeit zu erhalten und weiter auszubauen, sind umfangreiche Anstrengungen erforderlich, die Visionen in praktische Lösungen umzusetzen. Die Frage der Wettbewerbsfähigkeit entscheidet sich jedoch nicht allein an den technischen und organisatorischen Möglichkeiten der Industrie 4.0 sondern an deren effizienten und produktivitätssteigernden sowie kostengünstigen Anwendung in den Unternehmen. Gerade das 
Verhältnis von befürchtetem Aufwand und nicht eindeutig abzuschätzenden Ergebnissen lässt Unternehmen noch zögern. Gebraucht werden sehr schnell überzeugende Lösungen in vielen Industriezweigen, um noch bestehende Zweifel auszuräumen. Betrachtet man die Möglichkeiten der Smart Factory mit cyber-physischen Systemen ist - insbesondere in der Metall- und Elektroindustrie mit ihren verzweigten, strategisch ausgerichteten KundenLieferanten-Beziehungen und den Erfahrungen bei der Gestaltung hocheffektiver Produktionssysteme - der weitere Weg vorgezeichnet, um wettbewerbsfähige Arbeit in Deutschland zu halten.

\section{Arbeitsorganisation und -gestaltung in der Smart Factory}

Der Mensch bleibt auch in der künftigen Smart Factory der entscheidende Produktionsfaktor. Damit bleibt auch der die jeweiligen historisch-konkreten technischen, organisatorischen und sozialen Bedingungen von Arbeitsprozessen berücksichtigende Auftrag an die Arbeitswissenschaft bestehen.

Auf diesen Sachverhalt weist Schütte - einer der Autoren der „Umsetzungsempfehlungen für das Zukunftsprojekt Industrie 4.0“ - hin, indem er ausführt, dass das Innovationshandeln sich nicht allein auf die Bewältigung technischer Herausforderungen konzentrieren darf, sondern immer auch konsequent auf eine intelligente Organisation der Arbeit und die Fähigkeiten der Beschäftigten (Kagermann et al. 2013, S. 56). Mit Industrie 4.0 werden die Potenziale der Mitarbeiter eine zentrale Rolle bei der Gestaltung und Anwendung sowie der wirtschaftlichen Nutzung der cyber-physischen Systeme spielen. Die Art und Weise, wie die einzelnen Mitarbeiter im Rahmen ,offener, virtuell gestalteter Arbeitsplattformen und umfassender Mensch-Maschine- und Mensch-System-Interaktionen“ wirksam sind, wird Änderungen erfahren. Dennoch bleiben die Mitarbeiter in ihrer Gesamtheit die Träger der planenden, steuernden, dispositiven, ausführenden usw. Tätigkeiten. Mit Sicherheit wird es Auswirkungen auf die Arbeitsinhalte, die Arbeitsaufgaben, die Arbeitsprozesse und Umgebungsbedingungen geben. In welcher konkreten Ausprägung und mit welchen Erfordernissen in Bezug auf die Gestaltung der Arbeitsbeziehungen dies geschehen wird, wird sich erst in den vielfältigen praktischen Anwendungsfällen zeigen. Ebenso ist davon auszugehen, dass sich die Anforderungen an die fachliche, räumliche und zeitliche Flexibilität der Beschäftigten anders gestalten.

Die Assistenzsysteme, das veränderte Zusammenwirken von Mensch und Technik in neuen Formen der Kommunikation, Interaktion und Kooperation, die sich daraus ergebende Arbeitsstrukturierung sowie das erforderliche lebenslange Lernen zur Beherrschung der sich ebenfalls ständig verändernden cyber-physischen Systeme schaffen Voraussetzungen, die körperliche und geistige Leistungsfähigkeit der Beschäftigten zu erhalten und ständig weiter zu entwickeln. Leistungsfähigkeit hängt einerseits von verschiedenen menschlichen Eigenschaften - wie Konstitution und Gesundheit - und Fähigkeiten ab, die der Mensch durch das eigene Verhalten, d. h. den Umgang mit sich selbst, entscheidend beeinflussen kann. Dazu gehört nicht nur die persönliche Lebensführung, sondern zum 
Beispiel auch das lebenslange Lernen. Heute ist allgemein anerkannt, dass die individuelle Produktivität der Menschen nicht vom kalendarischen, sondern vom biologischen Alter abhängt. Menschen altern unterschiedlich, die Leistungsstreuung unter Gleichaltrigen nimmt mit dem Alter zu, und die physische und psychische Leistungsfähigkeit wandelt sich im Laufe des Lebens. Es gibt Fähigkeiten, die mit zunehmendem Alter tendenziell abnehmen, z. B. Muskelkraft, Sehvermögen; Fähigkeiten, die eher gleich bleiben, z. B. Sprachkompetenz (Ausdrucksfähigkeit); und Fähigkeiten, die sich tendenziell verbessern, z. B. Sozialkompetenz, Beurteilungsvermögen. Unternehmen und Beschäftigte beeinflussen die Richtung und Geschwindigkeit dieser Veränderungen stark. Andererseits bietet die Gestaltung der Arbeit vielfältige Einflussmöglichkeiten, um die körperliche und geistige Leistungsfähigkeit zu erhalten und zu steigern. In Bezug auf Industrie 4.0 ist das z. B. über die Aufteilung von Arbeitstätigkeiten durch Interaktion und Kooperation zwischen Mensch und Technik, den Einsatz von Assistenzsystemen, möglich. Sie lassen gestaltbare Arbeitsbedingungen, Arbeitsinhalte, Arbeitszeitsysteme, usw. zu, die gewährleisten, die Leistungsfähigkeit der Beschäftigten langfristig zu erhalten und zu entwickeln. Möglich wird dies z. B. durch Befreiung von monotonen oder schweren Tätigkeiten, Belastungswechsel oder lebenslanges Lernen. Welche Möglichkeiten in den künftigen cyberphysischen Systemen der einzelnen Unternehmen genutzt werden (können), hängt stark von betrieblichen Bedingungen ab. Die Auswirkungen der technischen Assistenzsysteme sowie die Art und Weise des Zusammenwirkens von Mensch und Technik sind per se nicht allein von der Technik determiniert. Die konkrete betriebliche Gestaltung des Einsatzes und des Zusammenwirkens sind Ergebnisse der betrieblich konzipierten und umgesetzten Prinzipien der Arbeitsorganisation und Arbeitsgestaltung. Allgemein anerkannte arbeitswissenschaftliche Grundsätze und Erkenntnisse sowie Regelungen des Arbeitsschutzes sind hierbei eine selbstverständliche Grundlage. Darüber hinaus beeinflussen betriebliche, demografische, marktbezogene, wirtschaftliche und technologie- und konstruktivproduktbezogene Erfordernisse die Gestaltungsprozesse.

Mit der Gestaltung cyber-physischer Systeme eng verbunden ist das Ziel, die Flexibilität in Bezug auf Kundenerwartungen und -wünsche zu erhöhen. Das setzt neben flexibler Technik voraus, dass sowohl die Betriebs- als auch die Arbeitszeit flexibel bezüglich der Kapazitätsbedarfe ist. Dazu bedarf es einer längerfristigen Begleitforschung, die insbesondere die durch Fernsteuerung, Fernbedienung und Fernwartung gegebenen Möglichkeiten in die Untersuchung mit einbezieht.

\section{Arbeitsinhalte und Anforderungen an Aus- und Weiterbildung}

Die Arbeitsinhalte und Arbeitsaufgaben der Mitarbeiter werden sich in Industrie 4.0 verändern. Damit entstehen veränderte, teilweise völlig neue Anforderungen an Können, Fähigkeiten, Fertigkeiten und Kompetenzen der Beschäftigten. Aus heutiger Sicht wird das insbesondere Komplexitäts-, Abstraktions- und Problemlösungsanforderungen sowie Anforderungen nach selbstgesteuertem Handeln, kommunikativen Kompetenzen und Fähig- 
keiten zur Selbstorganisation betreffen (vgl. Ebenda S. 57). Diese veränderten Anforderungen bedürfen der qualifikationsseitigen Vorbereitung seitens der Beschäftigten. Neben traditionellen werden verstärkt arbeitsplatznahe, auch selbstorganisierte Qualifizierungsformen erforderlich sein. Das Lernen im Prozess der Arbeit einschließlich der Entwicklung geeigneter Instrumente und Module wird die Qualifizierungsstrategien in den Unternehmen nachhaltig prägen. Mehr als heute wird sich die Notwendigkeit des lebenslangen Lernens verstärken.

Das Zusammenwirken von Mensch und Technik sowie die Anwendung von Assistenzsystemen in cyber-physischen Systemen müssen nicht in allen Fällen die beschriebenen veränderten Anforderungen bewirken. Die Autoren der Umsetzungsempfehlungen beschreiben auch die folgende mögliche Entwicklung: ,Je mehr das technische Integrationsniveau ansteigt, desto stärker könnten eine zunehmende Flexibilisierung, Intensivierung sowie eine steigende Spannung zwischen Virtualität und eigener Erfahrungswelt Raum greifen. Der Verlust an Handlungskompetenz, die Erfahrung der Entfremdung von der eigenen Tätigkeit durch eine fortschreitende Dematerialisierung und Virtualisierung von Geschäfts- und Arbeitsvorgängen wären die Folgen.“ (Ebenda)

Es ist aus heutiger Sicht schwerlich mit Sicherheit vorherzusagen, welche der beiden Stränge oder auch beide zusammen die Entwicklung in den Unternehmen bestimmen werden. Auch hier gilt, die konkreten Erfordernisse bestimmen die Lösungsansätze. Einfache Fortschreibung heutiger Lösungen in die Zukunft greifen sicher zu kurz. Der Hinweis auf die zwei potenziell konträren Linien:

- höhere Komplexitäts-, Abstraktions- und Problemlösungsanforderungen

- eventueller Verlust an (notwendiger?) Handlungskompetenz

zeigt zugleich Chancen für die künftige Gestaltung der Arbeitsinhalte: Auf der einen Seite Arbeitsaufgaben mit hohen, vielgestaltigen Anforderungen (technisch, technologisch, betriebswirtschaftlich, IT-spezifisch usw.) und auf der anderen einfachere Aufgaben, die in einer modernen Arbeitswelt weiterhin Chancen für gering Qualifizierte bieten. Wie sich diese Linien quantitativ gestalten, wird die weitere Entwicklung zeigen. Hier ist den Autoren der Umsetzungsempfehlungen zuzustimmen, die ausführen: „Die Technik bietet Optionen in beide Richtungen. Die Systemauslegung kann sowohl als restriktive, kontrollierende Mikrosteuerung, als auch als offenes Informationsfundament konfiguriert werden, auf dessen Basis der Beschäftigte entscheidet. Anders gesagt: Über die Qualität der Arbeit entscheiden nicht die Technik oder technische Sachzwänge, sondern Wissenschaftler und Manager, welche die Smart Factory modellieren und umsetzen.“ (Ebenda). Einflüsse darauf haben viele heute schon bekannte Faktoren wie z. B. das demografisch determinierte Angebot an Fachkräften, internationale Kooperationsbeziehungen, Kostenstrukturen, Flexibilisierungserfordernisse und -möglichkeiten usw.

Industrie 4.0 wird die Ansprüche an lebenslanges Lernen nicht allein auf die Industrie und deren Systeme der Aus- und Weiterbildung erhöhen. Auch in der akademischen Ausbildung und Weiterqualifizierung wird dies seinen Niederschlag finden müssen. Es ist zu 
erwarten, dass die Zusammenarbeit von Entwicklern und Anwendern langfristig die heutigen Grenzen zwischen IuK-Technologien, Produktions- und Automatisierungstechnik und Software verwischt. Das wird einen Prozess erfordern, der sich mit der weiteren Entwicklung von Industrie 4.0 immer schärfer stellt.

„Die Vielfalt der möglichen Einsatzgebiete setzt einer standardisierten Ausbildung Grenzen. Immer wichtiger wird der Dialog mit der produzierenden Industrie, um die Anforderungen der digitalen Ökonomie in die Ausbildung aufzunehmen. Unternehmen werden damit zukünftig noch stärker als heute zu Bildungspartnern von Hochschulen. An ein komprimiertes Erststudium müssen sich Einsätze in der betrieblichen Praxis und Vertiefungsstudien anschließen. Dabei gilt es, die Grenzen zu den Natur- und Ingenieurswissenschaften zu öffnen und überfachliche Kompetenzen wie Management oder Projektsteuerung stärker zu adressieren.“ (Ebenda).

Die relevanten Lerninhalte für Industrie 4.0 - sowohl was die akademische Ausbildung als auch die berufsbezogene Aus- und Weiterbildung anbelangt - lassen sich heute nur allgemein beschreiben, wie es die Autoren der Umsetzungsempfehlungen tun. Es ist daher erforderlich, die Bildungsinhalte ständig mit den Erfordernissen abzugleichen und dabei auch den Bedarf an Überblickswissen und Verständnis für das Zusammenspiel aller Akteure im Produktionsprozess im Blick zu halten. Nicht vergessen werden sollte dabei, dass auch unter den Bedingungen der cyber-physischen Systeme einfache Arbeitsaufgaben bestehen, für die eine Einweisungen oder ein kurzzeitiges Anlernen ausreichend ist.

\section{Cyber-physische Systeme versus Produktionssysteme}

Die Gestaltung von cyber-physischen Systemen ist ohne gut funktionierende Produktionssysteme mit großer Wahrscheinlichkeit mit erheblichen Reibungs- und Effizienzverlusten verbunden. Produktionssysteme als ganzheitliche Managementsysteme ,sind stets auf die Erreichung der Unternehmensziele fokussiert. Betrachtet werden alle Aktivitäten über die gesamte Wertschöpfungskette, einschließlich der Lieferanten und Kunden.“ (Krichel et al. 2013).

Die Herausforderung für die Betriebe besteht darin, die Elemente und Methoden im Rahmen der Produktionssysteme auf die betriebsspezifischen Anforderungen ständig neu abzustimmen und zu hinterfragen. Die Erfahrungen bei der Implementierung von Produktionssystemen gilt es bei der Entwicklung und dem Einsatz von cyber-physischen Systemen zu nutzen. Wichtig ist, erst die Abläufe zu analysieren, Verschwendungen aufzudecken und zu beseitigen, und danach Standards für die Arbeitssysteme und Prozesse zu definieren, bei Veränderungen der Bedingungen zu überprüfen und ggf. anzupassen.

Die Produktionssysteme sollen in cyber-physischen Systemen selbständig auf ungeplante Ereignisse reagieren (Kagermann et al. 2013, S. 108). „Im Zusammenwirken intelligenter Automatisierung mit der Erfahrung und der Kreativität von Menschen werden organisatorische Verluste in der Produktion sukzessive verringert. Zu diesem Zweck werden Produktionsarbeitern über ein mobiles Assistenzsystem kontextintensiv Informationen über die aktuellen Leistungsdaten der Produktion als Entscheidungsgrundlage für 
eine kontinuierliche Optimierung bereitgestellt.“ (Ebenda). Mit cyber-physischen Systemen entsteht demnach kein Konkurrenzsystem mit eigenen Regeln zu den heutigen Produktionssystemen, sondern ein mit zusätzlichen Entscheidungshilfen angereichertes und damit effektiveres. Damit wird deutlich, dass bereits mit den heutigen Aktivitäten zur Implementierung von Produktionssystemen wesentliche Voraussetzungen dafür geschaffen werden, die mit der Industrie 4.0 potenziell vorhandenen Möglichkeiten zur Sicherung und Steigerung der Wettbewerbsfähigkeit der Unternehmen zu erschließen.

Open Access This chapter is distributed under the terms of the Creative Commons Attribution Noncommercial License, which permits any noncommercial use, distribution, and reproduction in any medium, provided the original author(s) and source are credited.

\section{Literaturverzeichnis}

Kagermann, H., Wahlster, W., \& Helbig, J. (Hrsg.) (2013). Deutschlands Zukunft als Produktionsstandort sichern - Umsetzungsempfehlungen für das Zukunftsprojekt Industrie 4.0 - Abschlussbericht des Arbeitskreises Industrie 4.0. April 2013 http://www.hightech-strategie.de/files/ Umsetzungsempfehlungen_Industrie4_0.pdf.

Krichel, U., Reichel, F.-G., Neuhaus, R., \& GESAMTMETALL, ifaa (Hrsg.) (2013). Neuausrichtung der betrieblichen Organisation auf ein Produktionssystem. Gestaltungstechnische und arbeitsrechtliche Einführungshinweise für M+E-Betriebe. Köln: IW Medien. 